\title{
BÉZIER REPRESENTATION OF THE CONSTRAINED DUAL BERNSTEIN POLYNOMIALS
}

\author{
STANISEAW LEWANOWICZ^ AND PAWEŁ WOŹNY
}

\begin{abstract}
Explicit formulae for the Bézier coefficients of the constrained dual Bernstein basis polynomials are derived in terms of the Hahn orthogonal polynomials. Using difference properties of the latter polynomials, efficient recursive scheme is obtained to compute these coefficients. Applications of this result to some problems of CAGD is discussed.
\end{abstract}

AMS classification: Primary 41A10. Secondary 65D17, 33D45

Keywords. Dual Bernstein basis; Constrained dual Bernstein basis; Bézier polynomial form; Jacobi polynomials; Hahn polynomials.

\section{INTRODUCTION}

Let $\Pi_{n}^{(k, l)}$, where $k+l \leq n$, be the space of all polynomials of degree $\leq n$, whose derivatives of order $\leq k-1$ at $t=0$, as well as derivatives of order $\leq l-1$ at $t=1$, vanish. In particular, $\Pi_{n}:=\Pi_{n}^{(0,0)}$ is the space of the unconstrained polynomials of degree at most $n$. Obviously, $\operatorname{dim} \Pi_{n}^{(k, l)}=n-k-l+1$, and the Bernstein polynomials $\left\{B_{k}^{n}, B_{k+1}^{n}, \ldots, B_{n-l}^{n}\right\}$, where

$$
B_{i}^{n}(x)=\left(\begin{array}{c}
n \\
i
\end{array}\right) x^{i}(1-x)^{n-i} \quad(0 \leq i \leq n),
$$

form a basis of this space. There is a unique dual constrained Bernstein basis of degree $n$,

$$
\left\{D_{k}^{(n, k, l)}(x ; \alpha, \beta), D_{k+1}^{(n, k, l)}(x ; \alpha, \beta), \ldots, D_{n-l}^{(n, k, l)}(x ; \alpha, \beta)\right\} \subset \Pi_{n}^{(k, l)},
$$

satisfying the relation $\left\langle D_{i}^{(n, k, l)}, B_{j}^{n}\right\rangle=\delta_{i j} \quad(i, j=k, k+1, \ldots, n-l)$, where $\delta_{i j}$ equals 1 if $i=j$ and 0 otherwise, and the inner product $\langle\cdot, \cdot\rangle$ is given by

$$
\langle f, g\rangle:=\int_{0}^{1}(1-x)^{\alpha} x^{\beta} f(x) g(x) \mathrm{d} x \quad(\alpha, \beta>-1) .
$$

The unconstrained dual basis $D_{i}^{n}(x ; \alpha, \beta)$ corresponds to the case $k=l=0$, hence $D_{i}^{n}(x ; \alpha, \beta)=$ $D_{i}^{(n, 0,0)}(x ; \alpha, \beta)$.

Dual Bernstein polynomials $D_{i}^{(n, k, l)}(x ; \alpha, \beta)$ were studied by Ciesielski [4] (case $\alpha=\beta=0$ and $k=l=0$ ), also by Jüttler [6] (case $\alpha=\beta=0$ and general $k=l$ ), Rababah and Al-Natour [11] $(k=l=0$ and general $\alpha, \beta)$ and [12] (general $\alpha, \beta$ and $k=l$ ).

In [8], we have given explicit expressions for $D_{i}^{n}(x ; \alpha, \beta)$ in terms of the shifted Jacobi polynomials $R_{j}^{(\alpha, \beta)}(x)$, which, as it is well known, form a set of orthogonal polynomials with

Date: February 28, 2022.

${ }^{\star}$ Corresponding author. 
respect to the inner product (1.1). See also (2.3). In [16], we show that the constrained polynomials $D_{i}^{(n, k, l)}(x ; \alpha, \beta)$ are related to the unconstrained dual Bernstein polynomials of degree $n-k-l$, with parameters $\alpha+2 l$ and $\beta+2 k$. Consequently, using the above-mentioned results of [8], we obtain an explicit orthogonal representation, as well as degree elevation formula for the constrained dual Bernstein basis polynomials. The latter result plays a crucial role in the algorithm of multi-degree reduction of Bézier curves, given in the cited paper.

In some computer-aided geometric design applications, like curve intersection using Bézier clipping (see, e.g., [15, 2, 10]), polynomial approximation of rational Bézier curves [9], or degree reduction of Bézier curves (see, e.g., [16] and the papers cited therein), it is desirable to have the Bézier-Bernstein representation of the dual constrained polynomials,

$$
D_{i}^{(n, k, l)}(x ; \alpha, \beta)=\sum_{j=k}^{n-l} C_{i j}(n, k, l, \alpha, \beta) B_{j}^{n}(x) .
$$

In this paper, we give an efficient algorithm to compute the coefficients $C_{i j}(n, k, l, \alpha, \beta)$ for $i, j=1,2 \ldots, n$, which has $\mathcal{O}\left(n^{2}\right)$ complexity. This should be compared to a rather complicated recursive algorithm given in [6] and its generalization of [12].

In the sequel, we use the notation

$$
(c)_{k}:=\prod_{j=0}^{k-1}(c+j) \quad(k \geq 0) .
$$

The generalized hypergeometric series is defined by (see, e.g., [1, §2.1], or [7, §1.4])

$$
{ }_{r} F_{s}\left(\begin{array}{c}
a_{1}, \ldots, a_{r} \\
b_{1}, \ldots, b_{s}
\end{array} \mid z\right):=\sum_{k=0}^{\infty} \frac{\left(a_{1}\right)_{k} \ldots\left(a_{r}\right)_{k}}{k !\left(b_{1}\right)_{k} \ldots\left(b_{s}\right)_{k}} z^{k},
$$

where $r, s \in \mathbb{Z}_{+}$and $a_{1}, \ldots, a_{r}, b_{1}, \ldots, b_{s}, z \in \mathbb{C}$.

\section{Dual Bernstein polynomials}

Bernstein polynomial basis of degree $n(n \in \mathbb{N})$ is given by

$$
B_{i}^{n}(x):=\left(\begin{array}{c}
n \\
i
\end{array}\right) x^{i}(1-x)^{n-i} \quad(0 \leq i \leq n) .
$$

Recall that

$$
R_{m}^{(\alpha, \beta)}(x):=\frac{(\alpha+1)_{m}}{m !}{ }_{2} F_{1}\left(\begin{array}{c|c}
-m, m+\alpha+\beta+1 & 1-x \\
\alpha+1 & 1-x
\end{array}\right.
$$

are the shifted Jacobi polynomials [13, p. 280, Eq. (46)]. For $\alpha>-1$ and $\beta>-1$, polynomials (2.1) are orthogonal with respect to the scalar product (1.1) (see, e.g., [13, p. 273]); more specifically,

$$
\left\langle R_{m}^{(\alpha, \beta)}, R_{n}^{(\alpha, \beta)}\right\rangle=\delta_{m n} B(\alpha+1, \beta+1) \frac{(\alpha+1)_{m}(\beta+1)_{m}}{m !(2 m / \sigma+1)(\sigma)_{m}} \quad(m, n \geq 0)
$$

with $\sigma:=\alpha+\beta+1$ and $B(\lambda, \mu)=\Gamma(\lambda) \Gamma(\mu) / \Gamma(\lambda+\mu)$. Recall that the Hahn polynomials are defined by [7, $\S 9.5]$

$$
Q_{m}(x ; \alpha, \beta, N):={ }_{3} F_{2}\left(\begin{array}{c|c}
-m, m+\alpha+\beta+1,-x & 1 \\
\alpha+1,-N & 1
\end{array}\right) \quad(m=0,1, \ldots, N ; N \in \mathbb{N}) .
$$


Bernstein polynomials have the following representation in terms of shifted Jacobi polynomial basis:

$$
B_{i}^{n}(x)=\left(\begin{array}{c}
n \\
i
\end{array}\right)(\alpha+1)_{n-i}(\beta+1)_{i} \sum_{j=0}^{n} \frac{(2 j+\sigma)(-n)_{j}}{(\alpha+1)_{j}(j+\sigma)_{n+1}} Q_{j}(i ; \beta, \alpha, n) R_{j}^{(\alpha, \beta)}(x),
$$

where $0 \leq i \leq n$. This result follows from a formula given in [14, using [1, Cor. 3.3.5]; see also [8, Eq. (5.4)]. Conversely, shifted Jacobi polynomials have the following representation in terms of Bernstein basis [3]:

$$
R_{i}^{(\alpha, \beta)}(x)=\frac{(\alpha+1)_{i}}{i !} \sum_{j=0}^{n} Q_{i}(n-j ; \alpha, \beta, n) B_{j}^{n}(x) \quad(0 \leq i \leq n) .
$$

Associated with the basis $B_{i}^{n}(x)$ there is a unique dual Bernstein polynomial basis of degree $n$,

$$
D_{0}^{n}(x ; \alpha, \beta), D_{1}^{n}(x ; \alpha, \beta), \ldots, D_{n}^{n}(x ; \alpha, \beta),
$$

defined so that

$$
\left\langle D_{i}^{n}, B_{j}^{n}\right\rangle=\delta_{i j} \quad(i, j=0,1, \ldots, n) .
$$

In [8], we gave a number of properties of polynomials $D_{i}^{n}(x ; \alpha, \beta)$, including the expansion

$$
D_{i}^{n}(x ; \alpha, \beta)=\frac{1}{B(\alpha+1, \beta+1)} \sum_{j=0}^{n}(-1)^{j} \frac{(2 j / \sigma+1)(\sigma)_{j}}{(\alpha+1)_{j}} Q_{j}(i ; \beta, \alpha, n) R_{j}^{(\alpha, \beta)}(x),
$$

where $0 \leq i \leq n$, as well as the following alternative formula representing the dual Bernstein polynomial $D_{i}^{n}(x ; \alpha, \beta)(0 \leq i \leq n)$ as a "short" linear combination of Jacobi polynomials with shifted parameters:

$$
D_{i}^{n}(x ; \alpha, \beta)=\frac{(-1)^{n-i}(\sigma+1)_{n}}{B(\alpha+1, \beta+1)(\alpha+1)_{n-i}(\beta+1)_{i}} \sum_{j=0}^{i} \frac{(-i)_{j}}{(-n)_{j}} R_{n-j}^{(\alpha, \beta+j+1)}(x) .
$$

In CAGD applications, it is desirable to express the dual basis polynomials in terms of the Bernstein basis. We have the following result.

Theorem 2.1. For $i=0,1, \ldots, n$, we have

$$
D_{i}^{n}(x ; \alpha, \beta)=\sum_{j=0}^{n} c_{i j}(n, \alpha, \beta) B_{j}^{n}(x),
$$

where the Bézier coefficients $c_{i j}(n, \alpha, \beta)(0 \leq j \leq n)$ are given by any of the following two forms:

$$
c_{i j}(n, \alpha, \beta):=\frac{1}{B(\alpha+1, \beta+1)} \sum_{m=0}^{n} \frac{(2 m / \sigma+1)(\beta+1)_{m}(\sigma)_{m}}{m !(\alpha+1)_{m}} Q_{m}(i ; \beta, \alpha, n) Q_{m}(j ; \beta, \alpha, n),
$$

$$
c_{i j}(n, \alpha, \beta):=\frac{(-1)^{n}(\sigma+1)_{n}(-\alpha-n)_{i}}{B(\alpha+1, \beta+1) n !(\beta+1)_{i}} \sum_{h=0}^{i} \frac{(-i)_{h}}{(-\alpha-n)_{h}} Q_{n-h}(n-j ; \alpha, \beta+h+1, n) .
$$


Proof. Formula (2.8) with $c_{i j}(n, \alpha, \beta)$ given by (2.9) (respectively, (2.10) ) follows by inserting expansion (2.4) in (2.6) (respectively, (2.7)) and doing some algebra.

Now, we show that the coefficients $c_{i j}(n, \alpha, \beta)(i, j=1,2 \ldots, n)$ given in Theorem 2.1 can be computed recursively, with a cost $\mathcal{O}\left(n^{2}\right)$.

Theorem 2.2. Quantities $c_{i j} \equiv c_{i j}(n, \alpha, \beta)$ defined in (2.9), (2.10) satisfy the following recurrence relation:

$$
\begin{aligned}
c_{i+1, j}=\frac{1}{A(i)}\{ & (i-j)(2 i+2 j-2 n-\alpha+\beta) c_{i j} \\
& \left.+B(j) c_{i, j-1}+A(j) c_{i, j+1}-B(i) c_{i-1, j}\right\},
\end{aligned}
$$

where $0 \leq i \leq n-1,0 \leq j \leq n$ and

$$
A(h):=(h-n)(h+\beta+1), \quad B(h):=h(h-n-\alpha-1) .
$$

The starting values are

$$
c_{0 j}=\frac{(\sigma+1)_{n}(\beta+2)_{n}}{n ! B(\alpha+1, \beta+1)} \cdot \frac{(-1)^{j}}{(\alpha+1)_{n-j}(\beta+2)_{j}} \quad(0 \leq j \leq n) .
$$

Proof. Observe that the expression on the right-hand side of (2.9) is the linear combination of products $Q_{m}(i ; \beta, \alpha, n) Q_{m}(j ; \beta, \alpha, n)$, with the coefficients independent of $i$ and $j$. Hence, the proof of the Theorem can be based on an idea similar to the one used in [16, Thm A6] to prove a recursion satisfied by the dual discrete Bernstein polynomials.

First, recall that the Hahn polynomials satisfy the difference equation (see, e.g., [7, §9.5]),

$$
\mathcal{L}_{x}^{N} Q_{m}(x ; \beta, \alpha, N)=m(m+\sigma) Q_{m}(x ; \beta, \alpha, N),
$$

where the operator $\mathcal{L}_{x}^{N}$ is given by

$$
\mathcal{L}_{x}^{N} y(x)=a_{N}(x) y(x+1)-c_{N}(x) y(x)+b_{N}(x) y(x-1)
$$

with

$$
\begin{gathered}
a_{N}(x):=(x-N)(x+\beta+1), \quad b_{N}(x):=x(x-\alpha-N-1), \\
c_{N}(x):=a_{N}(x)+b_{N}(x) .
\end{gathered}
$$

Now, using equation (2.9) we easily obtain that

$$
\begin{aligned}
\mathcal{L}_{i}^{n} c_{i j}= & \sum_{m=0}^{n} \frac{(2 m / \sigma+1)(\beta+1)_{m}(\sigma)_{m}}{B(\alpha+1, \beta+1) m !(\alpha+1)_{m}} \\
& \times m(m+\sigma) Q_{m}(i ; \beta, \alpha, n) Q_{m}(j ; \beta, \alpha, n) \\
= & \mathcal{L}_{j}^{n} c_{i j} .
\end{aligned}
$$

This implies equation (2.11).

By (2.10) and (2.2), the sum in $c_{0 j}$ reduces to ${ }_{2} F_{1}(j-n, n+\sigma+1 ; \alpha+1 ; 1)$ which can be summed by the Chu-Vandermonde formula (see, e.g., [7, (1.5.4)]). Hence follows (2.12). 
Remark 2.3. Rababah and Al-Natour [12] have given the formula

$$
\begin{aligned}
c_{i j}(n, \alpha, \beta)= & (-1)^{i+j}\left(\begin{array}{c}
n \\
i
\end{array}\right)^{-1}\left(\begin{array}{c}
n \\
j
\end{array}\right)^{-1} \\
& \times \sum_{h=0}^{\min (i, j)}(2 h+\beta+1)\left(\begin{array}{c}
n+\sigma+h \\
n+\beta+h+1
\end{array}\right)\left(\begin{array}{c}
n+\alpha-h \\
n-h
\end{array}\right)^{-1} v_{i h} v_{j h}
\end{aligned}
$$

with

$$
v_{m h}:=\left(\begin{array}{c}
n+\beta+h+1 \\
n-m
\end{array}\right)\left(\begin{array}{c}
n+\alpha-h \\
n+\alpha-m
\end{array}\right),
$$

which contains the earlier result of Jüttler [6] for $\alpha=\beta=0$.

\section{Constrained dual Bernstein polynomials}

Let $\Pi_{n}^{(k, l)}$, where $k+l \leq n$, be the space of all polynomials of degree $\leq n$, whose derivatives of order $\leq k-1$ at $t=0$, as well as derivatives of order $\leq l-1$ at $t=1$, vanish:

$$
\Pi_{n}^{(k, l)}:=\left\{P \in \Pi_{n}: P^{(i)}(0)=0(0 \leq i \leq k-1) ; P^{(j)}(1)=0(0 \leq j \leq l-1)\right\} .
$$

In particular, $\Pi_{n}:=\Pi_{n}^{(0,0)}$ is the space of the unconstrained polynomials of degree at most $n$. Obviously, $\operatorname{dim} \Pi_{n}^{(k, l)}=n-k-l+1$, and the Bernstein polynomials $\left\{B_{k}^{n}, B_{k+1}^{n}, \ldots, B_{n-l}^{n}\right\}$ form a basis of this space. There is a unique dual constrained Bernstein basis of degree $n$,

$$
D_{k}^{(n, k, l)}(x ; \alpha, \beta), D_{k+1}^{(n, k, l)}(x ; \alpha, \beta), \ldots, D_{n-l}^{(n, k, l)}(x ; \alpha, \beta),
$$

satisfying

$$
\left\langle D_{i}^{(n, k, l)}, B_{j}^{n}\right\rangle=\delta_{i j} \quad(i, j=k, k+1, \ldots, n-l) .
$$

Recently, we have shown that the polynomials $D_{i}^{(n, k, l)}$ can be expressed in terms of dual Bernstein polynomials without constraints. Namely, we have the following result.

Theorem 3.1 ([16]). For $i=k, k+1, \ldots, n-l$, the following formula holds:

$$
D_{i}^{(n, k, l)}(x ; \alpha, \beta)=U_{i} \cdot x^{k}(1-x)^{l} D_{i-k}^{n-k-l}(x ; \alpha+2 l, \beta+2 k),
$$

where

$$
U_{i}:=\left(\begin{array}{c}
n-k-l \\
i-k
\end{array}\right)\left(\begin{array}{l}
n \\
i
\end{array}\right)^{-1} .
$$

Now, using expansion (2.8) in (3.1), the following result is easily obtained.

Theorem 3.2. The constrained dual basis polynomials have the Bézier-Bernstein representation

$$
D_{i}^{(n, k, l)}(x ; \alpha, \beta)=\sum_{j=k}^{n-l} C_{i j}(n, k, l, \alpha, \beta) B_{j}^{n}(x) \quad(k \leq i \leq n-l),
$$

where

$$
C_{i j}(n, k, l, \alpha, \beta):=U_{i} U_{j} c_{i-k, j-k}(n-k-l, \alpha+2 l, \beta+2 k),
$$

notation used being that of (2.9) and (3.2). 
TABle 1 . The $C$-table

$\begin{array}{cccccc} & 0 & 0 & \ldots & 0 & \\ 0 & C_{k k} & C_{k, k+1} & \ldots & C_{k, n-l} & 0 \\ 0 & C_{k+1, k} & C_{k+1, k+1} & \ldots & C_{k+1, n-l} & 0 \\ \ldots \ldots \ldots \ldots \ldots \ldots \ldots \ldots \ldots \ldots \ldots \ldots \ldots \ldots & \ldots \ldots \ldots \\ 0 & C_{n-l, k} & C_{n-l, k+1} & \ldots & C_{n-l, n-l} & 0 \\ & 0 & 0 & \ldots & 0\end{array}$

Observe that the quantities $C_{i j}$ can be put in a square array (see Table 1).

Combining (3.4) with Theorem 2.2, we obtain the following theorem.

Theorem 3.3. Quantities $C_{i j} \equiv C_{i j}(n, k, l, \alpha, \beta)$ defined in (3.4) satisfy the recurrence relation

$$
\begin{aligned}
C_{i+1, j}=\frac{1}{A^{*}(i)}\{ & (i-j)(2 i+2 j-2 n-\alpha+\beta) C_{i j} \\
& \left.+B^{*}(j) C_{i, j-1}+A^{*}(j) C_{i, j+1}-B^{*}(i) C_{i-1, j}\right\},
\end{aligned}
$$

where $k \leq i \leq n-l-1, k \leq j \leq n-l$ and

$$
\begin{aligned}
& A^{*}(u):=(u-n)(u-k+1)(u+k+\beta+1) /(u+1), \\
& B^{*}(u):=u(u-n-l-\alpha-1)(u-n+l-1) /(u-n-1) .
\end{aligned}
$$

We adopt the convention that $C_{i j}:=0$ if $i<k$, or $i>n-l$, or $j<k$, or $j>n-l$. The starting values are

$$
C_{k j}=W \cdot \frac{(-1)^{j} U_{j}}{(\alpha+2 l+1)_{n-l-j}(k+\beta+2)_{j}} \quad(j=k, k+1, \ldots, n-l),
$$

where we use notation (3.2), and

$$
W:=\left(\begin{array}{l}
n \\
k
\end{array}\right)^{-1} \frac{(-1)^{k}(\sigma+2 k+2 l+1)_{n-k-l}(k+\beta+2)_{n-l}}{B(\alpha+2 l+1, \beta+2 k+1)(n-k-l) !} .
$$

Using equation (3.5), one may obtain the element $C_{i+1, j}$ in terms of four elements from the rows number $i$ and $i-1$ (see Table 2). Now, the $C$-table can be completed very easily in the following way.

TABLE 2. The cross rule

$$
\begin{array}{cc} 
& C_{i-1, j} \\
C_{i, j-1} & C_{i j} \quad C_{i, j+1} \\
& C_{i+1, j}
\end{array}
$$


BÉZIER REPRESENTATION OF THE CONSTRAINED DUAL BERNSTEIN POLYNOMIALS

Algorithm 3.4 (Computing the Bézier coefficients of the polynomials $D_{i}^{(n, k, l)}$ ).

(1) Compute quantities $C_{k k}, C_{k, k+1}, \ldots, C_{k, n-l}$, filling the first row of the $C$-table, by the formulas

$$
\begin{aligned}
C_{k, n-l} & :=\left(\begin{array}{l}
n \\
k
\end{array}\right)^{-1}\left(\begin{array}{l}
n \\
l
\end{array}\right)^{-1} \frac{(-1)^{n-k-l}(\sigma+2 k+2 l+1)_{n-k-l}}{B(\alpha+2 l+1, \beta+2 k+1)(n-k-l) !} \\
C_{k j} & :=\frac{(j-n)(j-k+1)(j+\beta+k+2)}{(j+1)(j-n+l)(j-\alpha-l-n)} C_{k, j+1} \\
& (j=n-l-1, n-l-2, \ldots, k) .
\end{aligned}
$$

(2) For $i=k, k+1, \ldots, n-l-1$ and $j=k, k+1, \ldots, n-l$, compute $C_{i+1, j}$, using the recurrence (3.5).

Remark 3.5. Obviously, in case $k=l=0$, the above scheme allows to compute the Bézier coefficients $c_{i j}(n, \alpha, \beta)$ of the unconstrained dual polynomial $D_{i}^{n}(x ; \alpha, \beta)(c f(2.8))$.

Remark 3.6. If $\alpha=\beta$ and $k=l$, we have the following symmetry property of the $C$-table:

$$
C_{i j}=C_{n-i, n-j} \quad(k \leq i, j \leq n-k) .
$$

This can be verified easily, using the definition (3.4) of $C_{i j}$ as well as the identity

$$
Q_{m}(n-i ; \alpha, \alpha, n)=(-1)^{m} Q_{m}(i ; \alpha, \alpha, n) .
$$

Thus, the cost of computing the $C$-table can be halved in this case.

Remark 3.7. Jüttler [6] studied the case of $k=l$ and $\alpha=\beta=0$ and gave a rather complex recurrence relation in $i$ and $k$, for the coefficients $c_{i, j}^{(k)}$ in

$$
D_{i}^{(n, k, k)}(x ; 0,0)=\sum_{j=k}^{n-k} c_{i, j}^{(k)} B_{j}^{n}(x) \quad(k \leq i \leq n-k) .
$$

Rababah and Al-Natour [12] extended his approach to the case of arbtitrary $\alpha, \beta>-1$.

\section{Applications}

Consider the following approximation problem. Given the function $f$ defined on $[0,1]$, we look for a polynomial $P_{m} \in \Pi_{m}$,

$$
P_{m}(t):=\sum_{i=0}^{m} p_{i} B_{i}^{m}(t)
$$

which gives minimum value of the squared norm

$$
\left\|f-P_{m}\right\|_{L_{2}}^{2}=\left\langle f-P_{m}, f-P_{m}\right\rangle
$$

with the constraints

$$
\left.\begin{array}{ll}
f^{(i)}(0)=P_{m}^{(i)}(0) & (i=0,1, \ldots, k-1), \\
f^{(j)}(1)=P_{m}^{(j)}(1) & (j=0,1, \ldots, l-1),
\end{array}\right\}
$$

where $k+l \leq m$.

This general model contains, for instance, 
(1) multi-degree reduction problem, where $f=L_{n}$ is the polynomial of degree $n, n>m$, given in Bézier form,

$$
L_{n}=\sum_{i=0}^{n} l_{i} B_{i}^{n}
$$

(see, e.g., [16] and references given therein);

(2) computing roots of polynomials by the method of clipping [15, 2, 10], which uses a specific variant of the multi-degree reduction with low value of the degree $m$, say, less than five;

(3) polynomial approximation of the rational Bézier form, where $f=R_{n}$,

$$
R_{n}=\frac{\sum_{i=0}^{n} \omega_{i} r_{i} B_{i}^{n}}{\sum_{i=0}^{n} \omega_{i} B_{i}^{n}}
$$

(see, e.g., [9] and references given therein).

Theorem 4.1. The coefficients $p_{0}, p_{1}, \ldots, p_{m}$ of the polynomial (4.1) minimising the error (4.2) with constraints (4.3) are given by

$$
\begin{aligned}
& p_{i}=\frac{(m-i) !}{m !} f^{(i)}(0)-\sum_{j=0}^{i-1}(-1)^{i+j}\left(\begin{array}{c}
i \\
j
\end{array}\right) p_{j} \quad(i=0,1, \ldots, k-1) ; \\
& p_{m-i}=(-1)^{i} \frac{(m-i) !}{m !} f^{(i)}(1)-\sum_{j=1}^{i}(-1)^{j}\left(\begin{array}{c}
i \\
j
\end{array}\right) p_{m-i+j} \quad(i=0,1, \ldots, l-1) ; \\
& p_{i}= \sum_{j=k}^{m-l} C_{i j}(m, k, l, \alpha, \beta)\left\langle f, B_{j}^{m}\right\rangle-\left(\sum_{j=0}^{k-1}+\sum_{j=m-l+1}^{m}\right) p_{j} K_{i j} \\
&(i=k, k+1, \ldots, m-l),
\end{aligned}
$$

where $C_{i j}(m, k, l, \alpha, \beta)$ are introduced in (3.3) and

$$
\begin{aligned}
K_{i j}= & \left(\begin{array}{c}
m \\
j
\end{array}\right)\left(\begin{array}{c}
m \\
i
\end{array}\right)^{-1} \frac{(-1)^{i-k}(k-j)_{m-k-l+1}}{(i-j)(i-k) !(m-l-i) !} \\
& \times \frac{(\alpha+l+1)_{m-j}(\beta+k+1)_{j}}{(\alpha+l+1)_{m-i}(\beta+k+1)_{i}} .
\end{aligned}
$$

Proof. Recall that (see, e.g., [5, p. 49])

$$
\begin{aligned}
& P_{m}^{(j)}(0)=\frac{m !}{(m-j) !} \sum_{h=0}^{j}(-1)^{j+h}\left(\begin{array}{l}
j \\
h
\end{array}\right) p_{h}, \\
& P_{m}^{(j)}(1)=\frac{m !}{(m-j) !} \sum_{h=0}^{j}(-1)^{j+h}\left(\begin{array}{l}
j \\
h
\end{array}\right) p_{m-j+h} .
\end{aligned}
$$

Using the above equations in (4.3), we obtain the forms (4.4) and (4.5) for the coefficients $p_{0}, p_{1}, \ldots, p_{k-1}$ and $p_{m-l+1}, \ldots, p_{m-1}, p_{m}$, respectively. 
The remaining coefficients $p_{i}$ are to be determined so that

$$
\left\|f-P_{m}\right\|_{L_{2}}^{2}=\left\|W-\sum_{i=k}^{m-l} p_{i} B_{i}^{m}\right\|_{L_{2}}^{2}
$$

has the least value, where

$$
W:=f-\left(\sum_{j=0}^{k-1}+\sum_{j=m-l+1}^{m}\right) p_{j} B_{j}^{m} .
$$

Remembering that $B_{i}^{m}$ and $D_{i}^{(m, k, l)}(k \leq i \leq m-l)$ are dual bases in the space $\Pi_{m}^{(k, l)}$, we obtain the formula

$$
p_{i}=\left\langle W, D_{i}^{(m, k, l)}\right\rangle=\sum_{j=k}^{m-l} C_{i j}(m, k, l, \alpha, \beta)\left\langle f, B_{j}^{m}\right\rangle-\left(\sum_{j=0}^{k-1}+\sum_{j=m-l+1}^{m}\right) p_{j} K_{i j},
$$

where

$$
K_{i j}:=\left\langle B_{j}^{m}, D_{i}^{(m, k, l)}\right\rangle .
$$

Formula (4.7) was obtained in 9]. Equation (4.6) now readily follows.

Remark 4.2. Evaluation of quantities $\left\langle f, B_{j}^{m}\right\rangle$, which are present in equation (4.6), is a simple task in case of multi-degree reduction or computing zeros by Bézier clipping, as we have

$$
\left\langle f, B_{j}^{m}\right\rangle=\left\langle L_{n}, B_{j}^{m}\right\rangle=\sum_{i=0}^{n} l_{i}\left\langle B_{i}^{n}, B_{j}^{m}\right\rangle,
$$

and

$$
\left\langle B_{i}^{n}, B_{j}^{m}\right\rangle=B(\alpha+1, \beta+1)\left(\begin{array}{c}
n \\
i
\end{array}\right)\left(\begin{array}{c}
m \\
j
\end{array}\right) \frac{(\alpha+1)_{n+m-i-j}(\beta+1)_{i+j}}{(\alpha+\beta+2)_{n+m}} .
$$

In case of approximation of the rational function, we have

$$
\left\langle f, B_{j}^{m}\right\rangle=\left\langle R_{n}, B_{j}^{m}\right\rangle=\sum_{i=0}^{n} \omega_{i} r_{i} I_{i j}
$$

where the integrals $I_{i j}:=\left\langle B_{i}^{n}, B_{j}^{m} / \sum_{h=0}^{n} \omega_{h} B_{h}^{n}\right\rangle$ should be computed using numerical methods (see, e.g., [9]).

\section{Conclusions}

We gave explicit formulae for the Bézier coefficients of the constrained dual Bernstein basis polynomials in terms of the Hahn orthogonal polynomials. Using difference equation satisfied by the latter polynomials, we obtained a recursive algorithm to compute efficiently these coefficients. Applications of this result to several problems is discussed, namely to the multi-degree reduction problem for the Bézier curves, computing roots of polynomials by the method of Bézier clipping and approximation of the rational Bézier curves by Bézier curves. 


\section{REFERENCES}

[1] G. E. Andrews, R. Askey, R. Roy, Special Functions, Cambridge Univ. Press, Cambridge, 1999.

[2] M. Bartoň, B. Jüttler, Computing roots of polynomials by quadratic clipping, Comput. Aided Geom. Design 24 (2007) 125-141.

[3] Z. Ciesielski, Explicit formula relating the Jacobi, Hahn and Bernstein polynomials, SIAM J. Math. Anal. 18 (1987) 1573-1575.

[4] Z. Ciesielski, The basis of $B$-splines in the space of algebraic polynomials, Ukrainian Math. J. 38 (1987) 311-315.

[5] G. E. Farin, Curves and Surfaces for Computer-Aided Geometric Design. A Practical Guide, third ed., Academic Press, Boston, 1996.

[6] B. Jüttler, The dual basis functions of the Bernstein polynomials, Adv. Comp. Math. 8 (1998) 345-352.

[7] R. Koekoek, P. Lesky, R. F. Swarttouw, Hypergeometric Orthogonal Polynomials and Their $q$-Analogues, Springer, Berlin, 2010.

[8] S. Lewanowicz, P. Woźny, Dual generalized Bernstein basis, J. Approx. Theory 138 (2006) 129-150.

[9] S. Lewanowicz, P. Woźny, P. Keller, Polynomial approximation of rational Bézier curves with constraints, submitted.

[10] L. Liu, L. Zhang, B. Lin, G. Wang, Fast approach for computing roots of polynomials using cubic clipping, Comput. Aided Geom. Design 26 (2009) 547-559.

[11] A. Rababah, M. Al-Natour, Weighted dual functionals for univariate Bernstein polynomials, Appl. Math. Comp. 186 (2007) 456-463.

[12] A. Rababah, M. Al-Natour, Weighted dual functions for Bernstein basis satisfying boundary conditions, Appl. Math. Comp. 199 (2008) 1581-1590.

[13] Y.L. Luke, The Special Functions and Their Approximations, Vol. I, Academic Press, New York, 1969.

[14] A. Ronveaux, A. Zarzo, I. Area, E. Godoy, Bernstein bases and Hahn-Eberlein orthogonal polynomials, Integral Transforms Spec. Funct. 7 (1998) 87-96.

[15] T.W. Sederberg, T. Nishita, Curve intersection using Bézier clipping, Comput. Aided Design 22 (1990) $538-549$.

[16] P. Woźny, S. Lewanowicz, Multi-degree reduction of Bézier curves with constraints, using dual Bernstein basis polynomials, Comput. Aided Geom. Design 26 (2009) 566-579.

Institute of Computer Science, University of WrocŁaW, ul. F. Joliot-Curie 15, 50-383 WrocŁaW, POLAND

E-mail address: $\{$ Stanislaw.Lewanowicz, Pawel.Wozny\}@ii.uni.wroc.pl 